\title{
Learning efficacy of explicit visuomotor sequences in children with attention-deficit/hyperactivity disorder and Asperger syndrome
}

\author{
Katsumi Watanabe $\cdot$ Hanako Ikeda $\cdot$ Masutomo Miyao
}

Received: 28 September 2009 / Accepted: 4 March 2010 / Published online: 26 March 2010

(C) The Author(s) 2010. This article is published with open access at Springerlink.com

\begin{abstract}
Developmental disorders such as attentiondeficit/hyperactivity disorder (ADHD) and Asperger syndrome (AS) are often associated with learning disabilities. This study investigated the explicit learning of visuomotor sequences in 17 ADHD children (mean age 12.1), 21 AS children (mean age 12.7), and 15 typically developing children (mean age: 12.3). The participants were required to explore a hidden sequence of button presses by trial and error and elaborate the learned sequence $(2 \times 10$ task: Hikosaka et al. 1996). The results indicated that although ADHD and AS children had a tendency of repeating the same errors and took longer to complete a sequence, both showed a degree and pattern of improvement in accuracy
\end{abstract}

Electronic supplementary material The online version of this article (doi:10.1007/s00221-010-2217-3) contains supplementary material, which is available to authorized users.

K. Watanabe $(\bowtie) \cdot$ H. Ikeda

Research Center for Advanced Science and Technology,

University of Tokyo, 4-6-1, Komaba, Meguro-ku,

Tokyo 153-8904, Japan

e-mail: kw@ fennel.rcast.u-tokyo.ac.jp

K. Watanabe

National Institute of Advanced Industrial Science and Technology,

Ibaraki, Japan

K. Watanabe

Japan Science and Technology Agency, Saitama, Japan

H. Ikeda

Japan Society for Promotion of Science, Tokyo, Japan

M. Miyao

Department of Development and Behavioral Pediatrics,

National Center for Child Health and Development, Tokyo, Japan and speed similar to that of typically developing children. These results suggest that the explicit learning of visuomotor sequence in ADHD and AS patients is largely unimpaired.

Keywords Visuomotor sequence learning .

Developmental disorder · Asperger syndrome .

Attention-deficit/hyperactivity disorder $\cdot$ Learning curve

\section{Introduction}

Developmental disorders impose various restrictions on individuals, even if they are not accompanied with severe mental or intellectual impairment, and they often concur with learning disorders. However, it is still unclear whether learning difficulties in people with different developmental disorders are caused by the same cognitive mechanisms. It has been suggested that dysfunction in procedural learning is a possible cause of various learning disorders (Nicolson and Fawcett 2007), because it would lead to ineptitude in the classroom and/or motivational setbacks in daily life. The present study examines whether the learning profiles of discrete visuomotor sequences in children with attentiondeficit/hyperactivity disorder (ADHD) and Asperger syndrome (AS) differ from that in typically developing children, in order to elucidate the specificity and/or commonality in this form of procedural learning.

ADHD is characterized by symptoms such as inattention, hyperactivity, and impulsivity that exceed the children's developmental level. AS falls on the higher functioning end of autism spectrum disorder (ASD), which is characterized by impairments in social communication, repetitive behavior, and restricted interests, without mental retardation or intellectual deficit. Previous studies have 
employed a variety of visuomotor procedural learning tasks, including sequential response time task (Mostofsky et al. 2000), rotary pursuit task (Gidley-Larson and Mostofsky 2008), and contextual cueing task and alternating sequential response time task (Barnes et al. 2008). Studies have shown that some individuals with ADHD and ASD displayed altered functioning of implicit visuomotor sequence learning. For example, implicit visuomotor sequence learning in children with high-functioning autism (HFA) differs from that in typically developing (TD) and ADHD children (Mostofsky et al. 2000; Gidley-Larson and Mostofsky 2008). On the other hand, Barnes et al. (2008) reported that the implicit learning of spatial contexts and temporal sequences was unimpaired in children with HFA and AS. It should be noted that while visuomotor procedural learning in children with the heterogeneous subtypes of ASD have been studied (occasionally in comparison with ADHD), few studies have examined visuomotor sequence learning exclusively with AS children. One of the purposes of the present study was to examine visuomotor sequence learning in AS children.

Another motive of the present study was to investigate visuomotor procedural learning in situations occurring in everyday life. Most procedural learning in daily life includes two stages: the early controlled exploration of (often hidden) patterns and the process of automatization after a pattern has been discovered (Anderson 1982). However, most studies till date have examined the implicit components of procedural learning and have not used a task that captures both early explicit exploration and subsequent automatization processes. In this study, we employed a sequential button press task (hereafter referred to as a $2 \times 10$ task), where participants were asked to find a hidden visuomotor sequence of discrete button presses and elaborate the learned visuomotor spatial sequence (Hikosaka et al. 1996; Sakai et al. 1998, 2003; Watanabe et al. 2006). In the $2 \times 10$ task, a sequence is learned through trial and error and therefore the exploration process (learning for accuracy) is explicit. Later, the discovered visuomotor sequence is elaborated through repetitive practice, which improves speed (i.e., the automatization process). We examined whether ADHD and AS children exhibited any differences in the explicit and implicit aspects of visuomotor sequence learning in the $2 \times 10$ task when compared with TD children.

\section{Method}

Participants

The participants were 17 children with ADHD (16 boys; mean age 12.1 years), 21 children with AS (19 boys; mean age 12.7 years), and 15 TD children (11 boys; mean age 12.3 years). There was no statistical difference in age (ANOVA; $F(2,50)=0.46, P=0.64$ ). For the ADHD and AS groups, those children who had been assessed previously and were currently attending the National Center for Child Health and Development, Japan, were selected. All the ADHD children met the criteria for either inattentivetype or combined-type ADHD in accordance with the Diagnostic and Statistical Manual, Fourth Edition (DSMIV-TR) (American Psychiatric Association 1994). They were also diagnosed by conducting semi-structured clinical interviews (ADHD Rating Scale-IV; DuPaul et al. 1998). The AS participants were diagnosed on the basis of the DSM-IV-TR criteria, and the diagnosis was confirmed by a trained pediatrician using Gillberg's criteria (Gillberg and Gillberg 1989; Gillberg 1991). ${ }^{1}$ The ADHD and AS children were kept off medication for a minimum of 2 days. The TD children were chosen by the patients' friends and partners. None of the TD children met the aforementioned clinical criteria for ADHD or AS. The procedure was approved by the internal review board of the National Center for Child Health and Development, and a written informed consent was given by all the children and/or their parents prior to the testing.

\section{Procedure}

The participants were trained to perform a $2 \times 10$ task (see supplementary Fig A). A panel with 16 LED buttons mounted on a $4 \times 4$ matrix was placed before a seated participant. The LED buttons were square-shaped $(10 \mathrm{~mm})$ with an 8-mm space between buttons. Another LED button at the bottom of the panel was used as the home key. The participants used their right index fingers to press the buttons. The home key was turned on at the beginning of each trial. When the participants pressed the home key for $500 \mathrm{~ms}, 2$ of the 16 target LEDs, which we call "set," were simultaneously illuminated. The participants were required to press the illuminated buttons in the correct order, which they discovered by trial and error. If they were successful, the LEDs got turned off successively and another pair of LEDs, a second set, was illuminated; the participants were required to press the second pair in the correct order. A total of 10 sets, which we call "hypersets," were presented in a fixed order for the completion of a trial. When the participants pressed a wrong button, all the LED

\footnotetext{
${ }^{1}$ Although the Autism Diagnostic Observation Schedule (Lord et al. 2000) and Autism Diagnostic Interview-Revised (Lord et al. 1994) are also widely used for diagnosis, these tests have not yet been fully implemented in Japan. Therefore, we did not perform additional testing; instead, we included their established clinical diagnoses in our study.
} 
buttons were briefly illuminated and the trial was aborted. The subjects then had to restart the trial beginning with the home key. A trial was considered successful when the participant completed an entire hyperset (10 sets). The same hyperset was repeated until the participant successfully completed 20 trials. The participants were asked to perform the task as quickly and as accurately as possible.

\section{Results}

We used two measures to assess the overall accuracy and speed of performance (e.g., Watanabe et al. 2006). As a measure of accuracy, we counted the number of trials performed by a participant to complete a hyperset (total error; TE). In order to evaluate speed, we measured the time that had elapsed from the moment the home key was pressed to the moment the second button of the final (10th) set was pressed for each successful trial (total time; TT). A significant decrease was found in both TE (Fig. 1a) and TT (Fig. 2a), indicating that learning occurred irrespective of the participant group. The TE rapidly decreased in the first few completed trials, whereas the TT decreased more gradually. These observations were similar to those in our previous studies (Hikosaka et al. 1996; Sakai et al. 1998, 2003; Watanabe et al. 2006). Two-way ANOVAs on TE showed the significant main effect for the factor of trial $(F(19,950)=73.06, P<0.01)$ but not for the participant group $(F(2,50)=1.04, P=0.36)$. The interaction was also significant $(F(38,950)=1.78, P<0.01)$. ANOVAs on TT indicated that the main effect was significant for both the trial $(F(19,950)=20.01, P<0.01)$ and the participant group $(F(2,50)=4.22, P=0.02)$, whereas this was not the case for the interaction $(F(38,950)=0.78, P=0.83)$.

TE accumulated both evitable repeated errors (pressing a wrong button despite having received feedback in the preceding trial) and inevitable errors (pressing a wrong button when faced with a particular set for the first time). We counted evitable repeated errors for each set within participant to find a particular set that the participant made most errors and took the number of errors in that set as the maximum number of repeated errors; we then calculated the ratio of the maximum repeated errors to the TE for each participant and averaged them (Fig. 1b). The repeated error rate was significantly different for each group $(F(2,50)=3.51, P<0.05)$. Tukey post hoc tests revealed a significant difference between the AS and TD children $(P<0.05)$.

TT is the total sum of individual response times for 10 correctly completed sets. Each action for a given set consisted of two components: the time taken to initially choose and press the correct button out of the two illuminated buttons (choice deliberation time: CDT) and the time to press
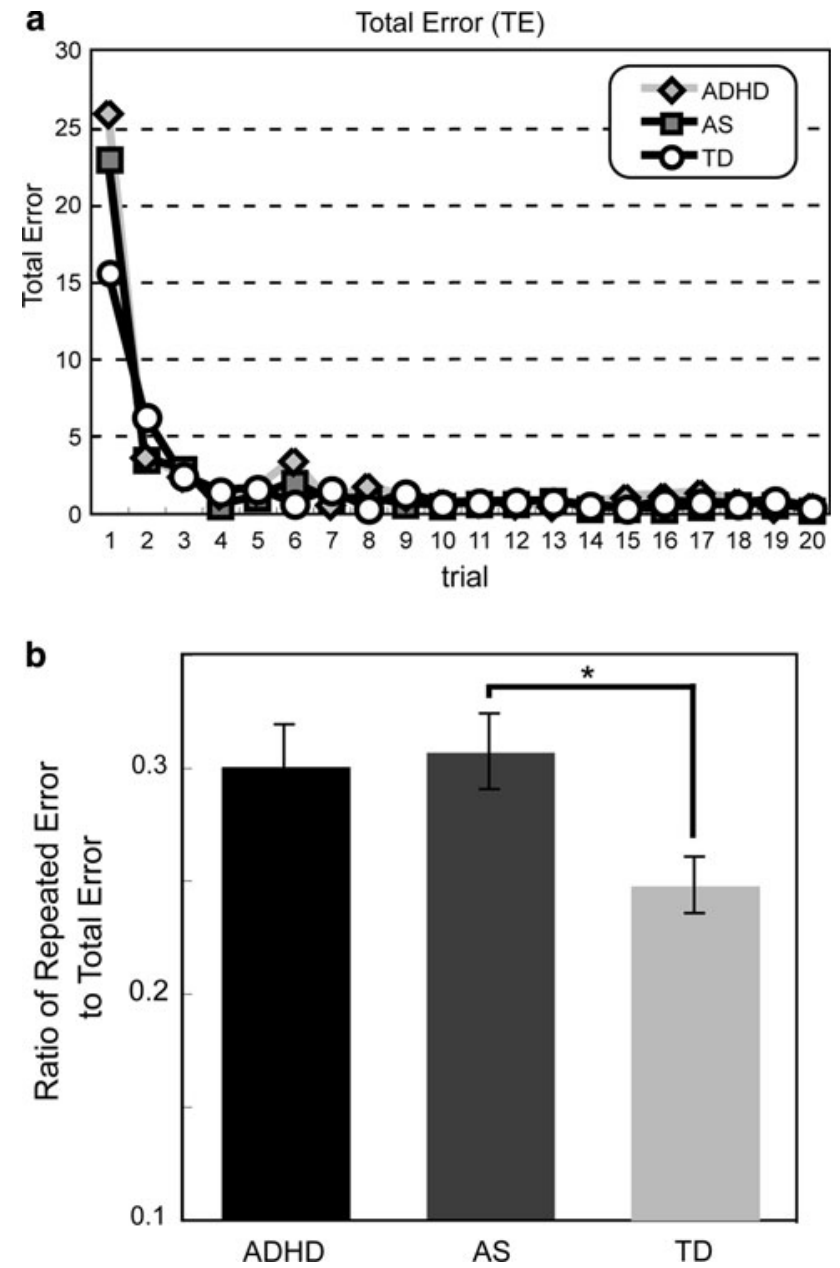

Fig. 1 a Averaged total errors (TEs) as a function of trial. TE decreased rapidly in the first few completed trials. b Averaged ratio of the maximum repeated errors to total errors, with bars indicating 1 SE. The repeated error rate tended to be higher for ADHD and AS children, but the difference reached the significant level only between AS and TD

the other button (simple response time: SRT) (supplementary Fig B). We calculated the sum of the CDT and SRT separately for each successful trial in order to examine how these two speed components would improve and contribute to the overall speed (Fig. 2b, c). In addition, to further elucidate the time course of learning, the TT, CDT, and SRT were fit with the power function (c.f., the power law of practice; Speelman and Kirsner 2005): $y=\alpha x^{-\beta}$, where $\alpha$ signifies the initial (and overall) performance level and $\beta$ represents the learning efficiency (shown in the inset of Fig. 2). The overall speed $(\alpha)$ seemed to be less for the ADHD and AS groups. Separate one-way ANOVAs showed that the difference was marginally significant for TT $(F(2,50)=2.69, P=0.08)$ and significant for CDT $(F(2,50)=3.35, P<0.05)$, but not for SRT $(F(2,50)=0.05$, $P=0.96)$. Further, Tukey post hoc tests revealed that this was because the ADHD group was significantly slower in 

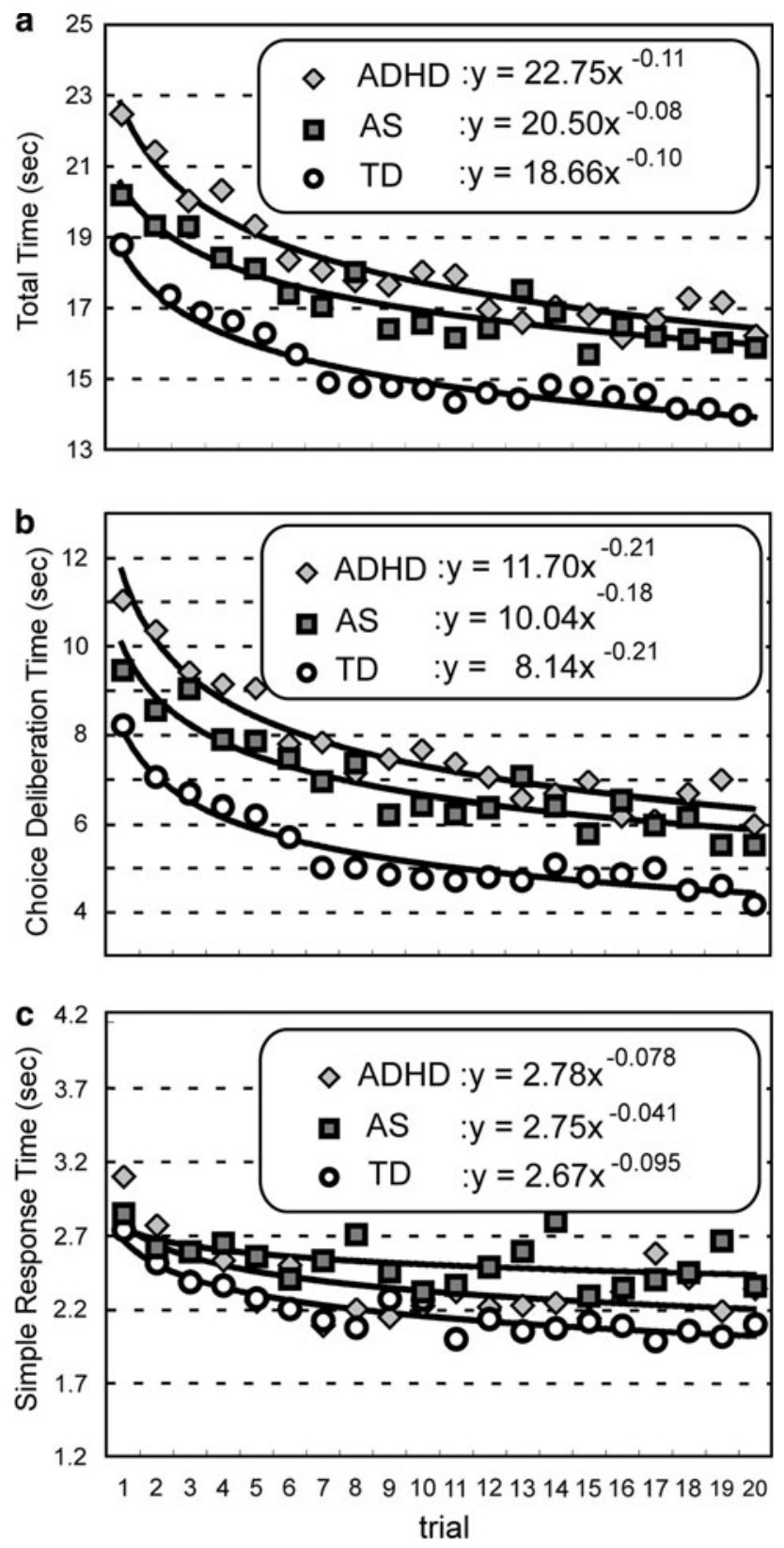

Fig. 2 a The time that had elapsed from the moment the home key was pressed to the moment the second button of the final set was pressed (total time; TT) as a function of trial, averaged for participants. TT decreased more gradually than TE. b The time to take to initially choose and press the correct button (choice deliberation time; CDT). c The time to then press the remaining button (simple response time; SRT). The data in (a-b) were fit with the power function: $y=\alpha x^{-\beta}$

the CDT than the TD group $(P<0.05)$. The learning efficiency or slope $(\beta)$ was not different among the groups $(F(2,50)=0.54, P=0.59$, for TT $F(2,50)=0.22, P=0.80$, for CDT: $F(2,50)=0.85, P=0.44$, for SRT). Thus, although the performance speed (particularly in CDT) was generally slower in the ADHD group, the learning process (reflected in $\beta$ ) appeared to be unaffected.

\section{Discussion}

This study examined whether the explicit learning of visuomotor sequence follows different patterns in children with ADHD and AS. The results showed the following: (1) With respect to speed improvement, both the ADHD and AS children showed patterns similar to those of the TD children (i.e., similar learning efficiencies, $\beta$ ). (2) The ADHD children showed significantly longer CDT (reflected in $\alpha$ ) than that of the TD children, and this value remained constant over the experimental session and contributed to enduring delays in completing the learned hypersets. (3) The AS children tended to make more repeated errors than the TD children.

Largely intact explicit visuomotor sequence learning in ADHD and AS

Mostofsky et al. (2000) suggested that visuomotor sequence learning in children with HFA may be different from that of TD and ADHD children. At the outset, this appears to be inconsistent with the present results. However, there are several differences between their study and the present one. First, our sample of ASD children consisted exclusively of AS patients, whereas their ASD patients included children with HFA. Barnes et al. (2008) reported that the implicit learning of spatial context and temporal sequences was unimpaired in their ASD participants, which consisted primarily of AS children (10 out of 14). Therefore, it is possible that distinct ASD cohorts show different learning profiles in visuomotor sequence learning (e.g., sequence learning may be affected in ASD children besides AS children). Second, Mostofsky et al. (2000) employed a simple sequential response time task, in which participants just need to follow a visual target. The $2 \times 10$ task requires participants to commit visuomotor sequences to memory. The difference in mnemonic demand with regard to performing the tasks might explain this discrepancy (e.g., a task demanding a mnemonic process may be more impervious to dysfunctions in AS children). Unimpaired implicit learning as found by Barnes et al. (2008) is consistent with this possibility; the complex spatial and temporal patterns might require more mnemonic processes in their task than in the simple sequential response time task. Third, the explicit aspect of the $2 \times 10$ task might lead to results different from those obtained by Mostofsky et al. (2000). Learning a visuomotor sequence in a practical setting often includes the explicit exploration of hidden patterns before the implicit automatization process. The $2 \times 10$ task had been devised to capture both processes, with more emphasis on the explicit exploration process; a complex visuomotor sequence is consciously explored and learned by trial and error, and elaborated once the sequence 
has been established. In addition, Gidley-Larson and Mostofsky (2008) found that, compared with both ADHD and TD children, HFA patients showed a similar degree of improvement as the ADHD patients in carrying out a rotary pursuit task; however, the degree of improvement was less when they needed to learn a pattern with another interference pattern. Thus, the preserved learning efficacy (i.e., similar degree of improvement) is common between their results and ours. In the present study, however, we did not ask the participants to learn multiple hypersets; therefore, we were unable to examine whether multiple hypersets would interfere with each other in the $2 \times 10$ task. These possibilities will form the basis of future investigations.

\section{Longer choice deliberation time}

The CDT appeared to be longer in both the ADHD and AS children than in the TD children. Indeed, the CDT in the ADHD children was significantly longer than in the TD children (Fig. 2b). The overall delay in the CDT is in accord with the previous finding of slower and variable processing speeds in ADHD children (Pennington and Ozonoff 1996; Leth-Steensen et al. 2000; McBurnett et al. 2001). There are at least two explanations for the prolonged CDT in ADHD. One is that the $2 \times 10$ task requires learning temporal patterns of sequential actions and ADHD children may experience difficulties in generating temporally organized sequences. It has been consistently found in various tasks that the responses of ADHD children are typically both slower and more variable than those of the control children (Douglas 1999; Leth-Steensen et al. 2000). Therefore, the $2 \times 10$ task, which requires the formation of temporally organized visuomotor sequences (e.g., chunking; Sakai et al. 2003) for smooth performance, would pose a problem for ADHD children. The other possibility is that ADHD children experience difficulties in resolving conflicting information. Studies have indicated that children with ADHD performed poorly when resolving conflicting information (Jonkman et al. 1999; Cairney et al. 2001; van Mourik et al. 2005; Johnson et al. 2008). The deficits in conflict resolution can be attributed to impairments in executive functions such as spatial working memory, set shifting, and the inhibition of proponent responses (Pennington and Ozonoff 1996; Barkley 1997; Tannock 1998; Douglas 1999; Wodka et al. 2007; Castellanos and Tannock 2002). Since the first button press of a given set requires participants to choose one of the two illuminated buttons, they need to avoid responding to the wrong button and choose the correct one. Through this task, a longer CDT in ADHD may reflect the additional time required for resolving conflicting information and/or responses. It should be noted, however, that these two possibilities are not exclusive, that is, they may even interact with each other.
Higher repeated error ratio

The repeated error ratio in the children with developmental disorders tended to be higher than in the TD children. In fact, the repeated error ratio was significantly higher in the AS children than in the TD children (Fig. 1b). This perseveration may reflect cognitive inflexibility and strict adherence to a routine, irrespective of the behavioral outcomes in ASD patients (including HFA and AS patients) (Ozonoff and Jensen 1999; Hill 2004). Since our results showed that the frequency of TE and SRT did not differ among the participant groups, it is likely that the deficit may not lie in a general perceptual-motor function but in inferior working memory or other executive functions. Executive dysfunctions are also found in ADHD children (e.g., Castellanos and Tannock 2002), yet, specific patterns of executive dysfunction that distinguish ASD (mainly planning) from ADHD (mainly inhibition) may continue to exist (Pennington and Ozonoff 1996; Sergeant et al. 2002; Hill 2004; Geurts et al. 2009). However, further investigation needs to be conducted to find out whether the observed repeated errors were due to executive dysfunction. Also it is important to examine whether the performance pattern is specific to AS children or generalized for other ASD children, because recent researches have indicated that AS children may have a different cognitive functioning and neurophysiological substrates when compared to other ASD children (Enticott et al. 2009; Sahyoun et al. 2009).

Limitations and future directions for research

Although the results indicated that the visuomotor sequence learning process in the $2 \times 10$ task is largely intact in ADHD and AS children, with some differences in the accuracy and speed patterns, the present study has several limitations. First, since the procedure was approved as a supplementary part of pediatric practice, there was a time restriction on single sessions, and this interfered with the testing of the effects of learning multiple hypersets. Second, the effect of extensive training was not tested; thus, it will be important to examine whether the observed difference in the CDT would persist even after extensive practice (more than 20 trials). Third, we admit that the diversities in ADHD and AS children (in relation to ASD) were not fully considered in this study (e.g., Geurts et al. 2009). Future research may include increasing the number of participants, implementing the administration of the Autism Diagnostic Observation Schedule (ADOS) or Autism Diagnostic Interview-Revised (ADI-R), and clearly classifying ADHD subtypes in order to confirm the present findings in a more homogeneous group. Finally, it would also be important to seriously consider the comorbidity between ADHD and AS. The current DSM-IV-TR states that ADHD and AS 
cannot be diagnosed simultaneously. However, recent investigations indicate that more than $40 \%$ of ASD children meet the symptom criteria for at least one of the three ADHD subtypes (Gadow et al. 2006; Willcutt et al. 2008). Similar learning curves between ADHD and AS children in the $2 \times 10$ task might arise from the presence of co-morbid ADHD/ASD. In addition, it is important to investigate the learning efficacy and general differences in patterns of errors and performance time in other pervasive developmental disorders (e.g., Rett syndrome), learning disorders (e.g., dyslexia), and developmental coordination disorders.

Despite these limitations and the fact that accepting the null hypothesis calls for caution, by employing the $2 \times 10$ task, which captures both explicit and implicit aspects in the procedural learning of relatively complex visuomotor sequence, and selecting ADHD and exclusively AS children as participants, the present results point to both commonality (i.e., similar learning efficiencies) among ADHD, AS, and TD children, and possible specificities (longer CDT in ADHD children and performance perseveration in AS children) for each group. As ADHD and AS are characterized by impaired functioning in multiple behavioral domains, unimpaired efficacy (which appear to be independent of executive dysfunctions) of visuomotor sequence learning would have implications for future research and treatment.

Acknowledgments This research was supported by Japan Science and Technology Agency, Japan Society for the Promotion of Science, MEXT Knowledge Custer Initiative (Toyama/Ishikawa Region), Grant-in-Aids for Scientific Research from the MEXT, and New Technology Foundation.

Open Access This article is distributed under the terms of the Creative Commons Attribution Noncommercial License which permits any noncommercial use, distribution, and reproduction in any medium, provided the original author(s) and source are credited.

\section{References}

American Psychiatric Association (1994) Diagnostic and statistical manual of mental disorders, 4th edn. American Psychiatric Association, Washington, DC

Anderson JR (1982) Acquisition of cognitive skill. Psychol Rev 89:369-406

Barkley RA (1997) Behavioral inhibition, sustained attention, and executive functions: constructing a unifying theory of ADHD. Psychol Bull 121:65-94

Barnes KA, Howard JH, Howard DV, Gilotty L, Kenworthy L (2008) Intact implicit learning of spatial context and temporal sequences in childhood autism spectrum disorder. Neuropsychol 22:563-570

Cairney S, Maruff P, Vance A, Barnett R, Luk E, Currie J (2001) Contextual abnormalities of saccadic inhibition in children with attention deficit hyperactivity disorder. Exp Brain Res 141:507-518

Castellanos FX, Tannock R (2002) Neuroscience of attention-deficit/ hyperactivity disorder: the search for endophenotypes. Nat Rev Neurosci 3:617-628
Douglas VI (1999) Cognitive control processes in attention deficit hyperactivity disorder. In: Quay HC, Hogan AE (eds) Handbook of disruptive behavior disorders. Plenum Press, New York, pp 105-138

DuPaul GJ, Power TJ, Anastopoulos AD, Reid R (1998) ADHD rating scale-IV: checklists, norms, and clinical interpretation. Guilford Press, New York

Enticott PG, Bradshaw JL, Iansek R, Tonge BJ, Rinehart NJ (2009) Electrophysiological signs of supplementary-motor-area deficits in high-functioning autism but not Asperger syndrome: an examination of internally cued movement-related potentials. Dev Med Child Neurol 51:787-791

Gadow KD, Devincent CJ, Pomeroy J (2006) ADHD symptom subtypes in children with pervasive developmental disorder. J Autism Dev Disord 36:271-283

Geurts HM, Corbett B, Solomon M (2009) The paradox of cognitive flexibility in autism. Trends Cog Sci 13:74-82

Gidley-Larson CL, Mostofsky SH (2008) Evidence that the pattern of visuomotor sequence learning is altered in children with autism. Autism Res 1:341-353

Gillberg C (1991) Clinical and neurobiological aspects of Asperger's syndrome in six families studied. In: Frith U (ed) Autism and Asperger's syndrome. Cambridge University Press, Cambridge, pp 122-146

Gillberg IC, Gillberg C (1989) Asperger syndrome-some epidemiological considerations: a research note. J Child Psychol Psychiatry 30:631-638

Hikosaka O, Sakai K, Miyauchi S, Takino R, Sasaki Y, Pütz B (1996) Activation of human presupplementary motor area in learning of sequential procedures: a functional MRI study. J Neurophysiol 76:617-621

Hill EL (2004) Evaluating the theory of executive dysfunction in autism. Dev Rev 24:189-233

Johnson KA, Robertson IH, Barry E, Mulligan A, Daibhis A, Daly M, Watchorn A, Gill M, Bellgrove MA (2008) Impaired conflict resolution and alerting in children with ADHD: evidence from the attention network task (ANT). J Child Psychol Psychiatry 49:1339-1347

Jonkman LM, Kemner C, Verbaten MN, van Engeland H, Kenemans JL, Camfferman G, Buitelaar JK, Koelega HS (1999) Perceptual and response interference in children with attention-deficit hyperactivity disorder, and the effects of methylphenidate. Psychophysiology 36:419-429

Leth-Steensen C, Elbaz ZK, Douglas VI (2000) Mean response times, variability, and skew in the responding of ADHD children: a response time distributional approach. Acta Psychol 104:167-190

Lord C, Rutter M, Le Couteur A (1994) Autism diagnostic interview revised: a revised version of a diagnostic interview for caregivers of individuals with possible pervasive developmental disorders. J Autism Dev Disord 24:659-685

Lord C, Risi S, Lambrecht L, Cook EH, Leventhal BL, DiLavore PC, Pickles A, Rutter M (2000) The autism diagnostic observation schedule-generic: a standard measure of social and communication deficits associated with the spectrum of autism. J Autism Dev Disord 30:205-223

McBurnett K, Pfiffner LJ, Frick PJ (2001) Symptom properties as a function of ADHD type: an argument for continued study of sluggish cognitive tempo. J Abnorm Child Psychol 29:207-213

Mostofsky S, Goldberg MC, Landa RJ, Denckla MB (2000) Evidence for a deficit in procedural learning in children and adolescents with autism: implications for cerebellar contribution. J Int Neuropsychol Soc 6:752-759

Nicolson RI, Fawcett AJ (2007) Procedural learning difficulties: reuniting the developmental disorders? Trends Neurosci 4:135-141

Ozonoff S, Jensen J (1999) Brief report: specific executive function profiles in three neurodevelopmental disorders. J Autism Dev Disord 29:171-177 
Pennington BF, Ozonoff S (1996) Executive functions and developmental psychology. J Child Psychol Psychiatry 37:51-87

Sahyoun CP, Soulieres I, Belliveau JW, Mottron K, Mody M (2009) Cognitive differences in pictorial reasoning between high-functioning autism and Asperger's syndrome. J Autism Dev Disord 39:1014-1023

Sakai K, Hikosaka O, Miyachi S, Takino R, Sasaki Y, Pütz B (1998) Transition of brain activation from frontal to parietal areas in visuomotor sequence learning. J Neurosci 18:1827-1840

Sakai K, Kitaguchi K, Hikosaka O (2003) Chunking during human visuomotor learning. Exp Brain Res 152:229-242

Sergeant JA, Geurts H, Oosterlaan J (2002) How specific is a deficit of executive functioning for attention-deficit/hyperactivity disorder? Behav Brain Res 130:3-28

Speelman CP, Kirsner K (2005) Beyond the learning curve: the construction of mind. Oxford University Press, Oxford, UK
Tannock R (1998) Attention deficit hyperactivity disorder: advances in cognitive, neurobiological, and genetic research. Child Psychol Psychiatry 39:65-69

van Mourik R, Oosterlaan J, Sergeant J (2005) The Stroop revisited: a meta-analysis of interference control in AD/HD. J Child Psychol Psychiatry 46:150-165

Watanabe K, Ikeda H, Hikosaka O (2006) Effects of explicit knowledge of workspace rotation in visuomotor sequence learning. Exp Brain Res 174:673-678

Willcutt EG, Sonuga-Barke EJS, Nigg JT, Sergeant JA (2008) Recent developments in neuropsychological models of childhood psychiatric disorders. Adv Biol Psychiatry 24:195-226

Wodka EL, Mahone EM, Blankner JG, Larson JC, Fotedar S, Denckla MB, Mostofsky SH (2007) Evidence that response inhibition is a primary deficit in ADHD. J Clin Exp Neuropsychol 29:345-356 